\title{
The Effects of Freezing on the Mechanical Properties of Bone
}

\author{
Bryan Kaye*, Connor Randall, Daniel Walsh and Paul Hansma
}

Department of Physics, University of California, Santa Barbara, California 93106, USA

\begin{abstract}
The serious health risks posed by bone fractures create a growing need for accurately diagnosing fracture risk. The Reference Point Indentation instrument (RPI) directly measures key mechanical properties in vivo to assess bone fracturability. There is a wealth of information that could be obtained from measuring cryopreserved bone samples with the RPI. Since it is unknown how freezing affects these key mechanical properties, measuring a cryopreserved sample gives no indication of the sample's original fracturability. Although there is research on how freezing affects the various mechanical properties of bone, this is the first paper to show how freezing effects the RPI's measurement of fracturability. Bovine femur and human tibia were tested using the RPI before freezing $\left(-20^{\circ} \mathrm{C}\right.$, up to 20 days $)$ and after thawing. The effect of freeze-thaw cycles varied depending on the type of the bone, but in most cases, was not measureable. When degradation did occur, the effect of freezing on the mechanical properties was smaller than the natural variation of those properties across a sample before freezing. Degradation of the mechanical properties, as measured by the RPI, was always found to be $15 \%$ or less. Subsequent freeze-thaw cycles had no effect on further degradation of the bone samples. In cases where degradation occurred, the effect from the twenty-day duration of freezing was negligible compared to the effects from phase-change. Furthermore, significant evidence was found supporting the theory that freezing degrades the organic components of the extracellular matrix.
\end{abstract}

Keywords: Cryopreservation, freezing, storage techniques, reference point indentation, bone diagnostic instrument, bone fracture.

\section{INTRODUCTION}

The serious health risks posed by bone fractures create a growing need for accurately diagnosing fracture risk. Applications of diagnosing fracture risk include determining the efficacy of osteoporosis medications. As the crucial work of Allen and Burr have shown, biopsies, which can be tested mechanically, are necessary to fully understand the effects of a medication on the fracturability of bone [1]. Currently available methods for directly testing the mechanical properties of bone require invasive bone sampling [2], making routine use in clinical settings unfeasible. In addition, a crucial report by Bouxsein shows that it is not clear which mechanical properties are most important to diagnosing fracture risk [3]. In a concerted effort to develop a reliable method to directly quantify fracture risk in living patients in a noninvasive manner, we have developed a new diagnostic instrument, the Reference Point Indentation Instrument [4] (RPI) (aka. Tissue Diagnostic Instrument [5], Bone Diagnostic Instrument [6-8], and Osteoprobe [9]) that uses a controlled indentation protocol to investigate the micro-mechanical response of the tibia to a defined loading regime. Clinical trials by Diez-Perez have shown the RPI to be successful at discriminating between patients with and without fragility fracture [4].

Preliminary results with the RPI have shown that an individual's susceptibility to fracture can be measured by

*Address correspondence to this author at the Hansma Lab UCSB Physics Department Santa Barbara, CA 93106, USA; Tel: (805) 893-3999;

Fax: (805) 893-8315; Email: bryankaye1@gmail.com indentation tests in which bone is forced, on a microscopic scale, into the same types of failure - separation of mineralized collagen fibrils - that is the root event of bone fractures. The RPI does this by using a probe assembly that can be inserted through the skin of a living patient and pressed up against the medial section of the tibia. The RPI can then measure the mechanical properties of bone by creating microscopic fractures in an indentation on the surface of the bone. The RPI obtains the displacement of the indenting probe as a function of the force applied and uses this information to calculate key parameters that are highly correlated with fracturability [4]. We believe that these parameters reflect the bone's ability to resist the growth of micro-cracks that can ultimately result in bone fracture.

With this novel instrument, we were able to study the effects of freezing on the mechanical properties of bone. Freezing is the preferred method for preserving bone used for transplantation [10] and thus understanding the effects of freezing is of crucial importance. At low temperatures, biological and biochemical processes slow down or stop completely [11]. Most enzymes of normothermic animals show a 1.5 to 2 fold decrease in metabolic rate for every $10^{\circ} \mathrm{C}$ decrease in temperature [11]. Many biological samples are preserved at low temperatures for these reasons [11]. Semen and embryos have been stored at cryogenic temperatures for years, then used successfully after thawing $[12,13]$. Generally speaking, subzero temperatures are used for preserving cells, whereas hypothermic temperatures are used for preserving organs [11]. Cowin suggests that $20{ }^{\circ} \mathrm{C}$ is an optimal temperature for bone preservation [10]. As for the effect of freezing on the mechanical properties of bone, 
previous experiments have found no difference after freezing $[14,15]$, while others have found significant differences after freezing $[16,17]$. These experiments incorporated a variety of mechanical tests, which include hardness, bending, and compression tests. As critical work by Schaffler et al has shown, discrepancies can stem from researchers' inability to characterize the full spectrum of mechanical properties that govern fracturability [18]. This is the first report on the effects of freezing using Reference Point Indentation, which provides measurements previously correlated to fracturability in vivo [4], therefore this is the first study to examine how freezing affects mechanical properties directly correlated to in vivo fracturability.

The extracellular matrix (ECM) is approximately $20 \%$ water by weight [19]. Water crystallization may create nanofractures in the cortical bone's extracellular matrix and thus may decrease bone strength [10]. This study is designed to quantify the effects from the duration of freezing, as well as to quantify the effects from phase change. Furthermore, we use the RPI to examine if freezing affects the organic part of the ECM differently than the inorganic part by comparing the effects of freezing on thermally degraded bone, which has a damaged organic ECM [20], to the effects of freezing on native bone.

\section{METHODS}

\subsection{Loading Regime and Key Measurements}

The RPI uses a custom hypodermic needle as a reference probe that rests on the surface of the bone. The test probe cycles in and out of the reference probe as it indents the surface of the bone. The loading on the test probe increases linearly from 0 to $11 \mathrm{~N}$ in $1 / 3$ of the indenting cycle. The load stays at $11 \mathrm{~N}$ for the next $1 / 3$ of the cycle. Then the load decreases linearly from 11 to $0 \mathrm{~N}$ for the last $1 / 3$ of the cycle. 20 indentation cycles are used per measurement at a rate of 2 $\mathrm{Hz}$.

Clinical trials reveal that large values of Indentation Distance Increase (IDI) and Total Indentation Distance (TID) are highly correlated with patients who have had bone fractures [4]. IDI is defined as the distance between the testprobe's displacement at maximum force on the $1^{\text {st }}$ cycle to the displacement at maximum force on the $20^{\text {th }}$ cycle. We hypothesize that this is a measure of how well the bone can resist the growth of a fracture. TID is defined as the total distance the test-probe indents into the bone. This may represent how well a bone can resist the start of a fracture as well as resist the growth of that fracture. We have chosen to omit unloading-slope data because we are uncertain of the relationship between unloading-slope and bone fracturability.

The same probe assembly was used to eliminate any possible systematic errors that are associated with changing probe assemblies in the middle of an experiment. A Zeiss Axioskop with 400x magnification was used in conjunction with a reticle to measure the probe radius before and after each testing session. The radius of the probe tip was always less than 2.5 microns. In addition to monitoring the tip radius, a calibration material, Polymethyl Methacrylate (PMMA), was measured before each testing over the course of the experiment to ensure proper instrument calibration.

\subsection{Sample Preparation}

Five bovine femur diaphyses were obtained from a local butcher. The bovine femur was de-fleshed and cut into approximately $3 \times 2 \mathrm{~cm}$ pieces. The pieces were mixed together and half of the pieces were selected at random for heating at $150^{\circ} \mathrm{C}$ for two hours (thermally degraded), while the other half were used for control. Since thermally degraded bone has a significantly weakened organic ECM [20], comparing the degradation of native bone to thermally degraded bone may give us insight into the effects of freezing on the organic ECM. Bovine bone was chosen for testing because its cortical bone is of the fibrolamellar type [10]. We hypothesize that this type of bone should exhibit greater changes due to freezing because of its woven bone layers, while still maintaining quintessential mechanical properties of cortical bone due to its laminar bone layers.

Two unfrozen human tibiae from women ages 71 and 83 were obtained from the University of California, Irvine (UCI) Willed Body Program. Although the exact date and time of death of the donors are unknown to us, the tissue had been preserved at hypothermic temperatures for up to an estimated four weeks before control tests were performed. The samples were kept hydrated but were not embalmed. Mechanical degradation may have occurred during this hypothermic storage period. Samples were obtained from the mid-shaft of the anterior tibia to simulate in vivo measurements, which are made on the tibia [9]. Samples were cut into approximately $3 \times 2 \mathrm{~cm}$ pieces.

All samples were frozen at $-20^{\circ} \mathrm{C}$. Samples were always hydrated from the moment they were de-fleshed by being gauzed wrapped in Hank's balanced salt solution (HBSS) [21] to ensure no degradation due to dehydration and to simulate in vivo conditions. Samples were thawed by placement in $22^{\circ} \mathrm{C}$ HBSS. Samples were tested in a small holding-vice that was placed in a plastic container filled with HBSS. The samples were placed lengthwise into the vice so that the flat (cut) ends were in contact with the vice plates.

All samples were sanded flat and then polished using a South Bay Technologies Model 900 polisher using sequentially finer polishing film followed by a 5-micron aluminum lapping film. All sanding and polishing was done under water flow and performed before freezing. The legitimacy of polishing bone samples was examined by measuring IDI and TID values of unpolished samples (periosteum removed), then polishing the samples and then re-measuring the IDI and TID values. It was found that measurements on the polished samples had approximately the same mean, but half the standard deviation of the unpolished samples. Polishing helped the study gain more statistical power, without increasing the sample size, by removing any irregularities and remaining soft tissue on the surface of the bone.

\subsection{Mechanical Testing on Bovine Femur}

Five native bovine bone samples were used. Four of the native bovine bone samples had a control test and a test after each freeze-thaw cycle (three freeze-thaw cycles in total). These samples were frozen for four days between tests. Each test is comprised of a row of 10 measurements (a single measurement gives both IDI and TID values). Each 
measurement was spaced 0.05 inches from the last measurement through the use of an X-Y translation stage. Rows of measurements are spaced at least 0.1 inches apart. Since the RPI indents are visible without the aid of optical instruments, spacing the rows was easy to accomplish with the X-Y translation stage.

To understand the effects from the duration of freezing, rather than the effects from phase change (freeze-thaw), we limited the freeze-thaw cycles to one, and varied the duration of freezing from four to twenty days. While four samples had a freeze-thaw test after four days, a fifth sample had a freeze-thaw test after twenty days. By comparing how the first four samples changed after the first freeze-thaw cycle (4 days in the freezer) to how this fifth sample changed after its first freeze-thaw cycle (20 days in the freezer), we can measure the effect from the duration of freezing.

Four thermally degraded bovine bone samples were used. Three thermally degraded bovine bone samples had one control test and a test after each freeze-thaw cycle (three freeze-thaw cycles in total). These thermally degraded bovine bone samples were frozen for four days between tests. The fourth thermally degraded bovine bone sample had one control test and one freeze-thaw cycle test after twelve days of being frozen. Again, this sample is used to measure the effect from the duration of freezing. There were ten measurements per row and each measurement was spaced by the same method used for the native bovine bone samples.

\subsection{Mechanical Testing on Human Tibia}

On each sample there were three rows of measurements made before the freeze-thaw cycle and three rows of measurements made after the freeze-thaw cycle. There were ten measurements per row and each measurement is spaced by the same method described in section 2.3. Rows extended lengthwise down the long axis of the bone sample. Control and freeze-thaw rows were staggered in an effort to offset the effect of variation in the bone's mechanical properties across the sample.

\subsection{Statistical Analysis}

For bovine bone experiments, the control test measurements from every sample were grouped together. Another group is made from combining all of the first freeze-thaw measurements, and another group from the second freeze-thaw measurements, etc. For analyzing the effect of freeze-thaw cycles, the control group was compared against every freeze-thaw group using a two-tailed, heteroscedastic t-test. For analyzing the effect of duration, the difference in IDI and TID measurements from control to the first freeze-thaw cycle of the samples frozen for 4 days were grouped together and was compared, using a twotailed, heteroscedastic t-test, to the difference in IDI and TID measurements from the control to the first freeze-thaw cycle from the sample frozen for 20 days. This statistical method was used for both native bovine bone and thermally degraded bovine bone. For human samples, the control test measurements from every sample (four samples, $n=120$ measurements) were grouped together and were compared, using a two-tailed heteroscedastic students test, to the freezethaw measurements from every sample (four samples, $n=120$

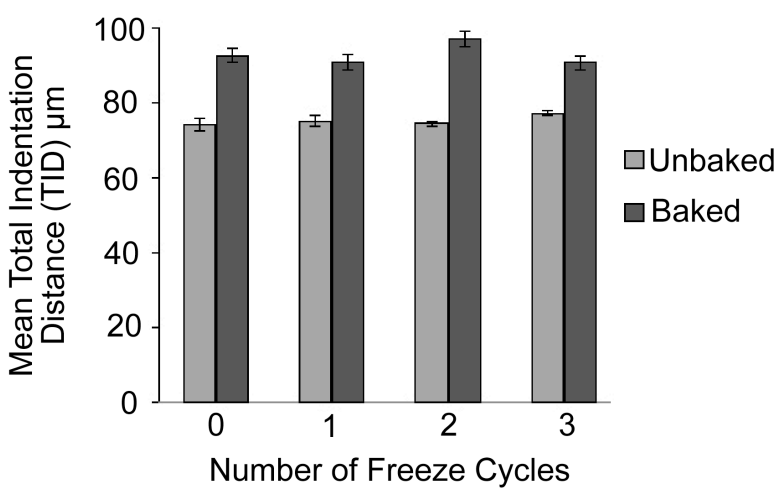

Fig. (1). Degradation of mechanical properties, as measured by TID, of thermally degraded compact bovine femur due to multiple phase-changes. Standard error is shown. Phase-change had no statistically significant effect on Total Indentation Distance on native $(\mathrm{p}=0.72,0.92,0.10)$ and thermally degraded bone $(\mathrm{p}=0.52$, $0.12,0.48$ ) from control to freeze-thaw cycle 1,2 , and 3 , respectively.

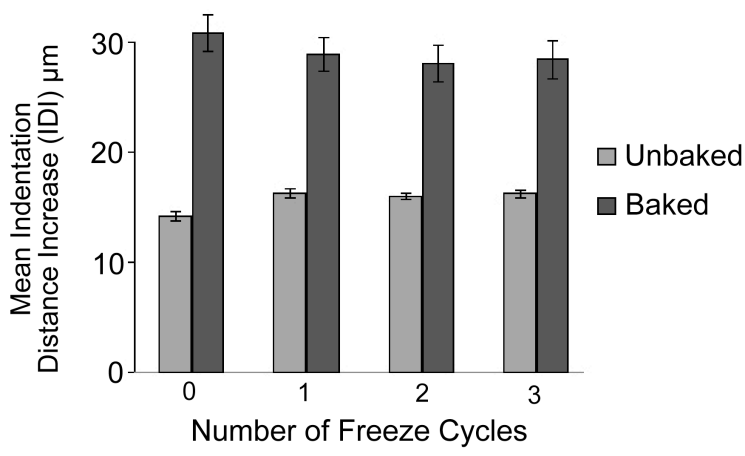

Fig. (2). Degradation of mechanical properties of compact bovine femur, as measured by IDI, of thermally degraded and native bone due to multiple phase-changes. Standard error is shown. Thermally degraded bone has no statistically significant change in IDI with multiple freeze-thaw cycles. Native compact bovine femur shows a minor degradation, the IDI increased by $15 \%$, after the first freezethaw cycle $(p=0.0008)$. Subsequent freeze-thaw cycles show no further statically significant degradation $(\mathrm{p}=0.52,0.94)$, as measured by IDI.

measurements). $\mathrm{P}$ values of 0.05 or less are considered statistically significant.

\section{RESULTS}

\subsection{Freeze-Thaw}

For native bovine bone, there was no statistically significant change in TID from freezing. TID measurements increased by $1.0 \%(p=0.72), 0.2 \%(p=0.92)$, and $4.0 \%(p$ $=0.10)$ between control and freeze-thaw cycle one, two and three, respectively (Fig. 1). IDI measurements increased by $15.1 \%(\mathrm{p}=0.0008)$ after one freeze-thaw cycle. The mean increased from 14.1 to 16.3 microns from control to first freeze-thaw cycle, with standard deviation 2.67 and 2.75 microns, respectively. Further freeze-thaw cycles had no statistically significant effect on IDI as IDI decreased by $1.9 \%(p=0.52)$ and $0.3 \%(p=0.94)$ between freeze-thaw cycle one and freeze-thaw cycle two and three, respectively (Fig. 2). 


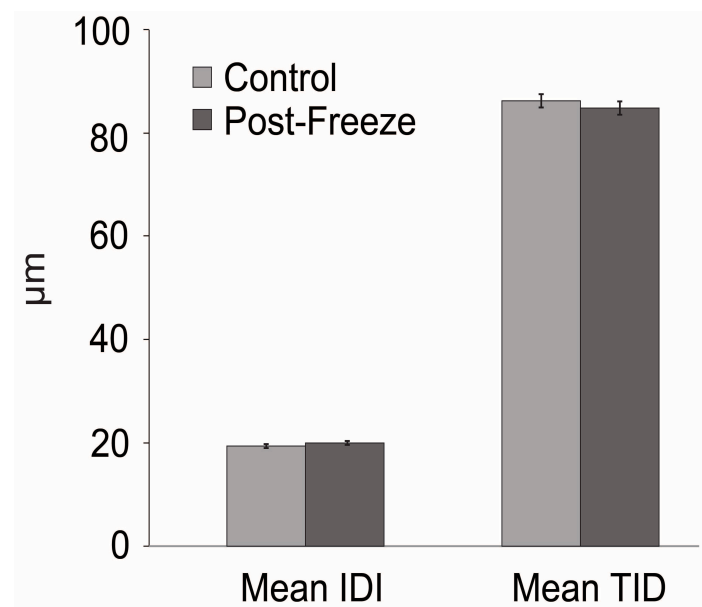

Fig. (3). Changes in mechanical properties of human tibia cortical bone, as measured by IDI and TID, due to freezing. Standard error is shown. Freezing caused IDI measurements increased by an average of $2.6 \%$ and TID decreased by an average of $2.6 \%$. The changes in IDI $(p=0.34)$ and TID $(p=0.42)$ were not statistically significant.

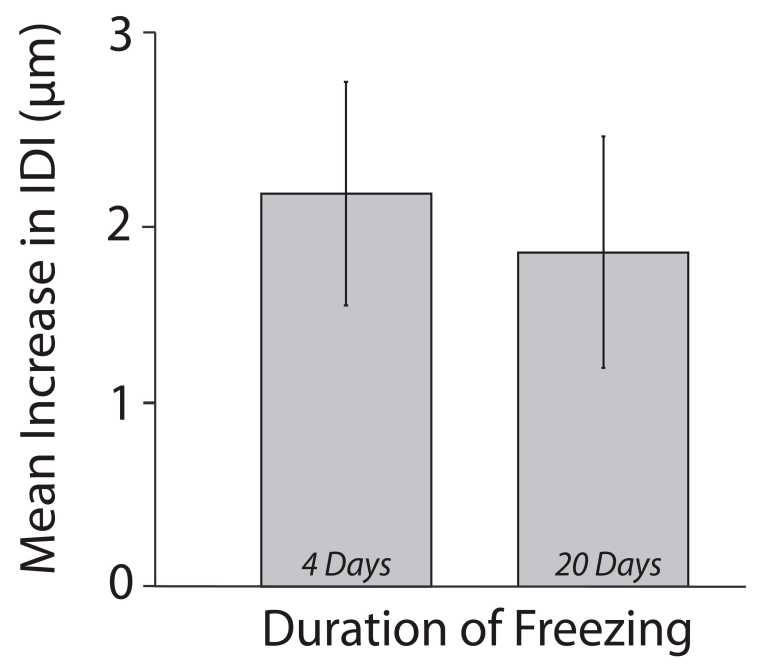

Fig. (4). Degradation of the mechanical properties of cortical bovine femur due to duration of freezing of as tested with the RPI. Measurements $(\mathrm{N}=10)$ from a bone samples frozen for five weeks at $-20{ }^{\circ} \mathrm{C}$ exhibited no statistically significant difference $(\mathrm{p}=0.74)$ in mechanical properties (as measured by IDI) compared to measurements from four samples frozen for 1 week $(\mathrm{N}=40)$ at the same temperature. Error bars represent standard error.

Human tibia showed an average $2.6 \%$ increase in IDI and an average $2.6 \%$ decrease in TID from control measurements to freeze-thaw cycle one measurements (Fig. 3). The changes in IDI $(\mathrm{p}=0.34)$ and in TID $(\mathrm{p}=0.42)$ were not statistically significant $(\mathrm{n}=105)$.

\subsection{Duration}

Measurements from native bovine bone samples that were frozen for four days $(\mathrm{n}=40)$ and twenty days $(\mathrm{n}=10)$ had a 15.1 and $16.3 \%$ increase in IDI, respectively, from control to freeze-thaw cycle one (Fig. 4). This difference in increases $(1.2 \%)$ of IDI between the two durations of freezing is minimal compared to the increase in IDI (15.1\%) due to phase-change on this time-scale. Furthermore, the difference in increases is not statistically significant $(\mathrm{p}=$ 0.74). Therefore all the changes in IDI are wholly or substantially due to phase change.

\subsection{Thermally Degraded Bone}

Freezing did not have a statistically significant effect on thermally degraded bovine bone. For IDI measurements, freeze-thaw cycles 1,2 , and 3 were not statistically different $(\mathrm{p}=0.38, \mathrm{p}=0.70, \mathrm{p}=0.84)$ from the control measurements. For TID measurements, freeze-thaw cycles 1 , 2 , and 3 were not statistically different $(\mathrm{p}=0.52, \mathrm{p}=0.12, \mathrm{p}$ $=0.48$ ) from the control measurements (Figs. 1, 2).

\section{DISCUSSION}

The RPI did not measure any statistically significant changes in IDI or TID due to freezing at $-20^{\circ} \mathrm{C}$ on the compact bone on the surface of human tibia, which is in close agreement with previous work. Research by Weaver found that freezing at $-20^{\circ} \mathrm{C}$ for 48 hours had no effect on the hardness of the cortical bone of human tibia [22]. Sedlin used bending to test human femoral cortical bone and found that freezing at $-20^{\circ} \mathrm{C}$ did not have a statistically significant effect on ultimate fiber stress, modulus of elasticity, or energy absorbed to failure [15]. Guidoni et al found that freezing at $-15^{\circ} \mathrm{C}$ did not affect the elastic modulus of compact bovine bone as measured by nanoindentation [23]. Cowen's survey of the effects of freezing on the mechanical properties of bone found that two thirds of the studies found no change with freezing, and that in most cases where changes were found, the samples were from rats and frozen at $-70^{\circ} \mathrm{C}$ or below [10].

While there was no statistically significant change in TID in native bovine bone, IDI had a statistically significant change $(15.1 \%, \mathrm{p}=0.0008)$ after the first freeze-thaw cycle. However, no degradation was seen in thermally degraded bovine bone. This is in close agreement with Cowen's summary of previous work on freezing, noting that when changes were seen, they were on the order of $10-25 \%$ [10]. This increase in fracturability is smaller than the natural variation across a sample (19\%), as calculated by the average standard deviation in IDI normalized by the mean IDI. Furthermore, previous results with RPI indicated that patients with fractures have, on average, a $47 \%$ larger IDI than patients that have not experienced fractures [4].

Measurements from a sample cryopreserved for longer periods of time showed similar degradation as measured using IDI, (16.3\%), to those preserved for a shorter period of time $(15.1 \%)$, and this difference was not statistically significant $(\mathrm{p}=0.37)$. This suggests that the degradation due to the duration of freezing was found to be negligible compared to the degradation due to phase-change on the time scale of 20 days. The post-freeze-thaw bone, or "dead bone," is most likely depleted of cellular and enzymatic activity and therefore the results agree strongly with theory. However, even if cellular and enzymatic activity remains after freezing, we would not expect much degradation on the time scale of 20 days because biological processes substantially slow down or completely stop at cryogenic temperatures [11]. "Dead bone" exhibited no further degradation after the first freeze-thaw cycle. 
It has been established that the heating of bone to $150^{\circ} \mathrm{C}$ significantly degrades the extracellular organic matrix [20]. When thermal degradation or freezing are applied to bovine bone individually, the bone has an increased fracturability as measured by IDI. Since it was found that freezing does not significantly alter the IDI of thermally degraded bone, but does alter the IDI of native bone, results from this study suggest the process of thermal degradation alters bone in the same way freezing does. It follows that freezing degrades the organic ECM. However, the degradation due to freezing was significant enough to be measured in bovine bone but not significant enough to be measured in human bone.

Bovine bone was chosen because its cortical bone is of the fibrolamellar type [10]. The fibrolamellar structure maintains quintessential mechanical properties of laminar cortical bone due to its laminar bone layers. However, it may have exhibited degradation because of it's woven bone layers, which have a higher water content [24]. Bovine bone degrading due to excess water content (as compared to cortical bone) would suggest that water crystallization is a primary cause for increased bone fracturability in cryopreserved bone.

Native bovine bone, after the first freeze-thaw cycle, no longer became more fracturable with consequent freezing and thawing as measured by the RPI. Results from this study suggest the organic ECM reaches saturation degradation due to phase change after a single freeze-thaw cycle (at least on the order of 5 freeze-thaw cycles). Minimal crystallization damage to the organic portion of the ECM, or the cortical bone as a whole, is expected considering the results of Weaver, Sedlin, and Guidoni et al., and noting that bone collagen is only barely soluble [20].

It is interesting to note that in native bovine bone, TID remained constant but IDI increased after freezing. Because of how IDI and TID are calculated, one can deduce that the probe displacement during the first indentation cycle is reduced after freezing. However, the total distance the probe moves after the final cycle is the same. This may suggest the ability of native bovine bone to resist crack propagation has been reduced after freezing. If this pattern is seen in future experiments, it will most likely be useful in discovering the length scale at which the crystallization is breaking up the ECM.

Significant degradation may have occurred during this hypothermic storage period or during the life to death transition. Brown et al found that within 96 hours postmortem, the elastic modulus decreased exponentially as it asymptotically reached $\sim 40 \%$ of the initial value [25]. The exact post-mortem effects on IDI and TID are unknown and therefore this study is investigatory and further work is necessary. However, frozen samples could still be used to qualitatively compare the effects of different pathologies on the mechanical properties of bone. Following hypothermic preservation, hydrated cryopreservation at $20{ }^{\circ} \mathrm{C}$ has very minimal effects on the mechanical properties of bone. Therefore researchers should strongly consider freezing as a suitable preservation technique.

\section{CONFLICT OF INTEREST}

The measurements reported here were done with prototype instruments, but Active Life Scientific, Inc. now produces a commercial version of this instrument, the Biodent. Paul Hansma is a member of Active Life Scientific, which sells the Biodent product line of Reference Point Indentation, RPI, instruments for research use only.

\section{ACKNOWLEDGEMENTS}

We thank NIH (R01GM065354) for supporting this work and the UCI Willed Body Program for providing bone samples. We thank Dan Bridges and James Weaver for enlightening discussions on bone material properties and for helping us prepare figures.

\section{REFERENCES}

[1] Allen M and Burr D. Mineralization, microdamage, and matrix: How bisphosphonates influence material properties of bone. Bone key Osteovision 2007; 4: 49-60.

[2] Seeman E, Delmas PD. Mechanisms of disease - Bone quality- The material and structural basis of bone strength and fragility. $\mathrm{N}$ Engl J Med 2006; 354: 2250-61.

[3] Bouxsein ML. Bone quality: where do we go from here? Osteoporos Int 2003; 14: S118-27.

[4] Diez-Perez A, Güerri R, Nogues X, et al. Microindentation for in vivo measurement of bone tissue mechanical properties in humans. J Bone Miner Res 2010; 25, 1877-85.

[5] Hansma PK, Yu H, Schultz D, et al. The tissue diagnostic instrument. Rev Sci Instrum 2009; 80: 054303.

[6] Hansma PK, Turner PJ and Fantner GE. Bone diagnostic instrument. Rev Sci Instrum 2006; 77: 075105.

[7] Randall C, Mathews P, Yurtsev E. The bone diagnostic instrument III: testing mouse femora. Rev Sci Instrum 2009; 80: 065108.

[8] Paul Hansma PT, Barney D, Eugene Y, et al., The bone diagnostic instrument II: Indentation distance increase. Rev Sci Instrum 2008; 79: 064303.

[9] Hansma PK. A new bone diagnostic instrument: the osteoprobe II. J Bone Miner Res 2007; 22: S226-7.

[10] Cowin SC. Bone mechanics handbook. USA: CRC Press 2001

[11] Rubinsky B. Principles of low temperature preservation. Heart Fail 2003; 8: 227-84.

[12] Child born with parent's semen stored for 22 years. 2008 [cited $7^{\text {th }}$ Sep, 2011]; Available from: http://www.planer.com/company/news/146-child-born-with-parents-semen-stored-for-22-years. html

[13] 'Twins' born 16 years apart. 2011 [cited $7^{\text {th }}$ Sep, 2010]; Available from: http://www.planer.com/company/news/324-twins-born-16years-apart.html

[14] Weaver JK. The microscopic hardness of bone. J Bone Joint Surg Am 1966; 48: 273-88.

[15] Sedlin ED. A rheologic model for cortical bone. A study of the physical properties of human femoral samples. Acta Orthop Scand Suppl 1965; (Suppl. 83): 1-77.

[16] Kang JS, Kim NH. The biomechanical properties of deep freezing and freeze drying bones and their biomechanical changes after in vivo allograft. Yonsei Med J 1995; 36: 332.

[17] Pelker RR, Friedlaender GE, Markham TC, et al. Effects of freezing and freeze-drying on the biomechanical properties of rat bone. J Orthop Res 1984; 1: 405-11.

[18] Akkus O, Polyakova-Akkus A, Adar F, Schaffler MB. Aging of microstructural compartments in human compact bone. J Bone Miner Res 2003; 18: 1012-9.

[19] Gray H. Gray's Anatomy: the Anatomical Basis of Clinical Practice. $39^{\text {th }}$ ed., Edinburgh; New York: 2005, Elsevier Churchill Livingstone. p. 1627.

[20] Danielsen CC. Age-related thermal stability and susceptibility to proteolysis of rat bone collagen. Biochem J 1990; 272: 697-701.

[21] Habelitz S, Marshall GW Jr, Balooch M, Marshall SJ. Nanoindentation and storage of teeth. J Biomech 2002; 35: 995-8.

[22] Weaver JK. The Microscopic Hardness of Bone. J Bone Joint Surg 1966; (48-A): 273

[23] Guidoni G, Swain M, Jager I. Nanoindentation of wet and dry compact bone: Influence of environment and indenter tip geometry on the indentation modulus. Philos Mag 2010; 90: 553-65. 
[24] Weinstein SL, Buckwalter JA, Turek SL. Turek's Orthopaedics: Principles and their Application. $5^{\text {th }}$ ed. Philadelphia: Lippincott 1994, xiv, 708, p. 8 of plates.
[25] Brown N, Saputa C, Black J. Young's modulus of living human bone. Trans Orthop Res Soc 1981; 6: 41.

(C) Kaye et al.; Licensee Bentham Open .

This is an open access article licensed under the terms of the Creative Commons Attribution Non-Commercial License (http://creativecommons.org/licenses/by$\mathrm{nc} / 3.0 /$ ), which permits unrestricted, non-commercial use, distribution and reproduction in any medium, provided the work is properly cited. 